\title{
Extracellular Fluid Accumulation Predicts Fluid Responsiveness after Hydroxyethyl Starch 70/0.5 Bolus Infusion during Major Abdominal Surgery
}

\author{
Takeshi Ide $^{1}$, Tsuneo Tatara ${ }^{2}$, Takahiko Kaneko ${ }^{2}$, Shinichi Nishi ${ }^{1}$ \\ ${ }^{1}$ Department of Intensive Care Medicine, Hyogo College of Medicine, Nishinomiya, Japan; ${ }^{2}$ Department of Anesthesiology, Hyogo \\ College of Medicine, Nishinomiya, Japan. \\ Email: ttatara@hyo-med.ac.jp
}

Received September $15^{\text {th }}, 2013$; revised October $16^{\text {th }}, 2013$; accepted October $30^{\text {th }}, 2013$

Copyright (C) 2013 Takeshi Ide et al. This is an open access article distributed under the Creative Commons Attribution License, which permits unrestricted use, distribution, and reproduction in any medium, provided the original work is properly cited.

\begin{abstract}
Objective: The purpose of this study was to test the hypothesis that extracellular fluid accumulation predicts fluid responsiveness after hydroxyethyl starch (HES) solution bolus infusion during major abdominal surgery. Methods: Twenty patients who underwent elective pancreaticoduodenectomy under general anesthesia were studied. Patients received $4 \mathrm{~mL} / \mathrm{kg}$ boluses of Ringer's acetate or 6\% HES 70/0.5 solution over $15 \mathrm{~min}$ in random order when urine output decreased below $1.0 \mathrm{~mL} / \mathrm{kg} / \mathrm{h}$. Stroke volume variation (SVV) and stroke volume index (SVI) were measured using the FloTrac $^{\mathrm{TM}} /$ Vigileo $^{\mathrm{TM}}$ system at pre-bolus, 15,30 , and $60 \mathrm{~min}$ after initiating bolus infusion. The percent change in pre-bolus extracellular fluid volume relative to that at the skin incision for arm ( $\left.\Delta V_{\mathrm{ECF}}\right)$ was measured by bioelectrical impedance. Prediction of fluid responsiveness (an increase in SVI of $\geq 5 \%$ ) by pre-bolus SVV or pre-bolus $\Delta V_{\mathrm{ECF}}$ was tested by calculating the area under the receiver operating characteristic curve (AUC). Results: Fluid bolus infusions in this study consisted of 61 Ringer's acetate infusions and 62 HES infusions. The best AUCs for identifying fluid responsiveness were seen with pre-bolus $\Delta V_{\mathrm{ECF}}$ for HES at $30 \mathrm{~min}$ and $60 \mathrm{~min}(\mathrm{AUC}=0.74, P=0.022$; AUC $=0.74, P=$ 0.0054 , respectively). Optimal threshold values of pre-bolus $\Delta V_{\mathrm{ECF}}$ for predicting fluid responsiveness were $6.5 \%$ for 30 min (sensitivity: $78 \%$, specificity: $58 \%$ ) and $7.7 \%$ for $60 \mathrm{~min}$ (sensitivity: $56 \%$, specificity: $76 \%$ ). Conclusion: Extracellular fluid volume predicts fluid responsiveness after HES solution bolus infusion during major abdominal surgery. Substantial fluid responsiveness is observed upon increased accumulation of extracellular fluids.
\end{abstract}

Keywords: Plasma Expanders; Blood Volume; Fluid Therapy; Abdominal Surgery

\section{Introduction}

Volume replacement with hydroxyethyl starch (HES) solution according to goal-directed fluid therapy has been recommended in major abdominal surgery [1-3]. However, fluid therapy aimed at maximizing cardiac output by fluid challenge can lead to frequent HES bolus infusions, which should be avoided due to potential adverse effects on renal and coagulation functions [4,5]. Moreover, HES solution may leak into the interstitial space with time due to capillary leakage arising from surgical injury [6], thereby leading to interstitial edema and delaying postoperative recovery [7].

While stroke volume variation (SVV) predicts fluid responsiveness for surgical patients [8], little is known about the effect of extracellular fluid volume on fluid res- ponsiveness. Continuous infusion of crystalloid solution during surgery monotonously increases interstitial fluid accumulation with time [9], leading to a fall in colloid osmotic pressure in the interstitium [10]. Given that HES induces volume expansion via a colloid osmotic effect, it is possible that, with increased extracellular fluid accumulation, HES extracts more fluid from the interstitial space to the intravascular space due to a larger gradient of colloid osmotic pressure across the capillary wall, thereby increasing fluid responsiveness. This information may help explore the most effective timing of HES infusion and thus prevent overdose of HES solution during major abdominal surgery.

Given the inherent difficulties of controlling extracellular fluid volume for patients undergoing surgery, we 
performed a longitudinal analysis of fluid responsiveness as assessed by stroke volume index (SVI) during longduration major abdominal surgery. We [11] and other groups $[12,13]$ have demonstrated that localized perioperative fluid accumulation in the extracellular fluid space can be quantitatively analyzed by segmental bioelectrical impedance analysis. The aim of this study was to test the hypothesis that extracellular fluid accumulation, as measured by bioelectrical impedance, can predict fluid responsiveness after HES solution bolus infusion during major abdominal surgery.

\section{Materials and Methods}

\subsection{Patients}

This study was approved by the Ethics Review Board of Hyogo College of Medicine and written informed consent was obtained from each patient after explaining the study. As data on the effect of extracellular fluid volume on fluid responsiveness were not available in the literature, this study was planned as a pilot study. We enrolled 21 consecutive American Society of Anesthesiologists (ASA) physical status 1 - 3 patients (age range, 20 - 80 years) who were scheduled for elective pancreaticoduodenectomy for cancer from February 2010 to July 2011. Exclusion criteria included cardiac arrhythmia, severe pulmonary disease, severe renal dysfunction, chronic use of diuretics, and an expected duration of surgery $<6 \mathrm{~h}$.

\subsection{Procedure}

All patients fasted from midnight on the night before surgery. On arrival to the operating room, Ringer's acetate solution (RA) was intravenously infused into a peripheral vein on the left hand at a rate of $2 \mathrm{~mL} / \mathrm{kg} / \mathrm{h}$ using an intravenous catheter. Anesthesia was induced by fentanyl, propofol and rocuronium. After tracheal intubation, anesthesia was maintained with sevoflurane and oxygen in air, together with a continuous infusion of remifentanil. Mechanical ventilation was performed with PEEP $5 \mathrm{~cm}$ $\mathrm{H}_{2} \mathrm{O}$ and tidal volume $8 \mathrm{~mL} / \mathrm{kg}$. Diuretics were not used during surgery.

Hypotension (i.e., systolic arterial blood pressure $<80$ $\mathrm{mmHg}$ ) was treated by intravenous administration of ephedrine in $4 \mathrm{mg}$ increments if heart rate (HR) was $<90$ beats/minute and phenylephrine in $0.1 \mathrm{mg}$ increments if HR was $>90$ beats/minute. Packed red blood cells were transfused when blood hemoglobin concentration was $<8$ - $9 \mathrm{~g} / \mathrm{dL}$. When massive bleeding (e.g., $30 \mathrm{~mL} / \mathrm{kg}$ ) occurred, fresh frozen plasma was provided.

\subsection{Fluid Therapy}

At the time of skin incision, the rate of RA infusion was increased to $4 \mathrm{~mL} / \mathrm{kg} / \mathrm{h}$ (i.e., basal fluid infusion). Urine output was monitored every hour from the time of skin incision. If urine output over a 1-hour period decreased below $1.0 \mathrm{~mL} / \mathrm{kg}, 4 \mathrm{~mL} / \mathrm{kg}$ fluid boluses of RA solution or $6 \%$ HES solution (HES 70/0.5, average molecular weight 70,000 , molar substitution 0.5 , Salinhes ${ }^{\circledR}$, Fresenius-Kabi Japan, Tokyo, Japan) were provided over 15 min instead of basal fluid infusion (Figure 1). After finishing bolus infusion, RA infusion was restarted at basal infusion rates (i.e., $4 \mathrm{~mL} / \mathrm{kg} / \mathrm{h}$ ). For each bolus infusion, the choice of RA or HES solution was randomized by a computerized random number generator. No vasopressors were administered during bolus infusion.

\subsection{Hemodynamic Monitoring}

A catheter was inserted into the right radial artery and connected to the FloTrac ${ }^{\mathrm{TM}} /$ Vigileo $^{\mathrm{TM}}$ system (ver. 3.02, Edwards Lifesciences, Irvine, CA, USA) to obtain SVV and SVI. Hemodynamic variables including mean arterial blood pressure (MAP), HR, SVV, and SVI were recorded.

\subsection{Bioelectrical Impedance Analysis}

Multifrequency segmental bioelectrical impedance analysis was conducted for the right arm every hour after skin incision. Body resistance and reactance were measured using a multifrequency bioimpedance analyzer (4200 Hydra, Xitron Technologies, San Diego, CA, USA). The resistance at zero frequency (corresponding to resistance for extracellular fluid, $R_{\mathrm{e}}$ ) was determined by performing

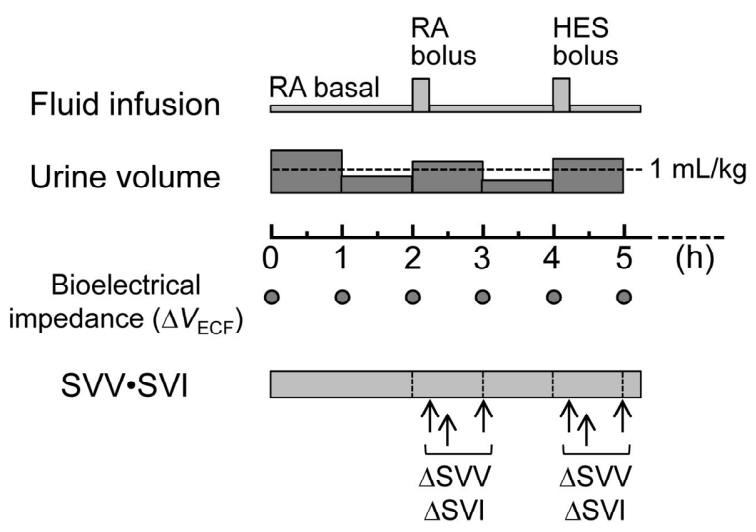

Figure 1. Study protocol. Patients received $4 \mathrm{~mL} / \mathrm{kg}$ boluses of Ringer's acetate (RA) or hydroxyethyl starch (HES) solution over $15 \mathrm{~min}$ in random order when urine output decreased below $1.0 \mathrm{~mL} / \mathrm{kg} / \mathrm{h}$. Time 0 denotes the time of skin incision. $\Delta V_{E C F}$ : percent change of extracellular fluid volume relative to that at skin incision; $S V V$ : stroke volume variation; SVI: stroke volume index; $\triangle S V V$ : change of SVV after start of bolus infusion relative to pre-bolus; $\Delta S V I$ : change of SVI after start of bolus infusion relative to prebolus. 
non-linear curve-fitting and subsequent extrapolation of impedance values at 50 frequencies from $5 \mathrm{kHz}$ to 1 MHz. Current-injecting electrodes were placed at the dorsal surface of the third metacarpal bone of the right hand and the center of the anterior face of the right humeral head.

The detector electrodes were placed at the anterior part of the right wrist and $5 \mathrm{~cm}$ distal to the center of the anterior face of the right humeral head. While high-frequency current passes through cell membranes and thus reflects total body fluid volume, low-frequency current passes only through extracellular fluid space due to cell membrane capacitance [14]. Based on the assumption that the electrical properties of body tissues are similar to those of a concentrated suspension of spherical particles (cells) in the outer conducting medium (extracellular fluid), the extracellular fluid volume for the arm ( $V_{\mathrm{ECF}}$, $\mathrm{mL} / \mathrm{kg}$ ) is related to the resistivity of the arm at zero frequency $(\rho, \mathrm{ohm} \cdot \mathrm{cm})[11,14]$ :

$$
V_{\mathrm{ECF}}=\frac{V}{W}\left(\frac{\rho_{\mathrm{ECF}}}{\rho}\right)^{2 / 3}
$$

where $V$ is the volume of the arm $\left(\mathrm{cm}^{3}\right), W$ is body weight $(\mathrm{kg})$, and $\rho_{\mathrm{ECF}}$ is the resistivity of extracellular fluid $(=50 \mathrm{ohm} \cdot \mathrm{cm})$. Given that the value of $\rho$ is proportional to $R_{\mathrm{e}}$ in the same patient, percent changes of $V_{\mathrm{ECF}}$ relative to baseline (i.e., at the skin incision) $\left(\Delta V_{\mathrm{ECF}}\right)$ is calculated as $\left(\left[R_{\mathrm{e}} \text { value relative to baseline }\right]^{-2 / 3}-1\right)$ multiplied by 100 .

\subsection{Data Analysis}

Pre-bolus (i.e., immediately before infusion) values for MAP, HR, and volume of urine produced during a 1-hour period from the start of infusion $(\mathrm{mL} / \mathrm{kg})$ were obtained. Pre-bolus $\Delta V_{\mathrm{ECF}}$ and changes in SVV (i.e., $\Delta \mathrm{SVV}$ in \%) and SVI (i.e., $\Delta$ SVI in \%) at $15 \mathrm{~min}, 30 \mathrm{~min}$, and $60 \mathrm{~min}$ after the start of infusion relative to pre-bolus were calculated.

\subsection{Statistical Analysis}

Data are expressed as mean (SD) or median (interquartile range) depending on the distribution. Time to bolus infusion (defined as hours from the time of skin incision to the time when the fluid bolus was started); pre-bolus values including MAP, HR, SVV, SVI, and $\Delta V_{\mathrm{ECF}}$; urine volume during a 1-hour period from the start of infusion; $\triangle \mathrm{SVV}$; and $\triangle \mathrm{SVI}$ were compared between RA and HES boluses using the Student's t-test or Mann-Whitney rank-sum test. Data for pre-bolus $\Delta V_{\mathrm{ECF}}$ for all fluid boluses were analyzed using linear regression, with prebolus SVV set as an independent variable. One-way repeated measures analysis of variance with the Student-
Newman-Keuls post hoc test was used to compare $\Delta$ SVI between $15 \mathrm{~min}, 30 \mathrm{~min}$, and $60 \mathrm{~min}$ after the start of infusion for RA and HES boluses.

Fluid responsiveness was defined as an increase in SVI of $\geq 5 \%$. Pre-bolus values of SVV and $\Delta V_{\mathrm{ECF}}$ were compared between non-responding boluses and responding boluses for RA and HES. The prediction of fluid responsiveness by pre-bolus SVV or pre-bolus $\Delta V_{\mathrm{ECF}}$ was tested by calculating the area under the receiver operating characteristic curves (AUCs) with 95\% confidence intervals (CIs). Threshold values for pre-bolus SVV and pre-bolus $\Delta V_{\mathrm{ECF}}$ were determined to maximize both sensitivity and specificity. GraphPad Prism 5 (GraphPad Software Inc., San Diego, CA, USA) and SigmaPlot 12 (Systat Software Inc., Chicago, IL, USA) were used for statistical analysis. $P<0.05$ was considered statistically significant.

\section{Results}

\subsection{Patient Characteristics}

One patient was excluded from the analysis because the surgical procedure was changed to a palliative surgery that lasted $<6 \mathrm{~h}$. Patient characteristics and intraoperative parameters are shown in Table 1. Fresh frozen plasma was provided to two patients $(7.3 \mathrm{~mL} / \mathrm{kg}$ and 14.8 $\mathrm{mL} / \mathrm{kg}$ ).

\subsection{Comparison of RA and HES Bolus Infusion Parameters}

We performed a total of 123 fluid bolus infusions (RA: $n$

Table 1. Patient characteristics and intraoperative parameters.

\begin{tabular}{cc}
\hline Variable & $\mathrm{N}=20$ \\
\hline Gender (male/female) & $13 / 7$ \\
Age $(\mathrm{yr})$ & $67(11)$ \\
Weight $(\mathrm{kg})$ & $56(10)$ \\
Body mass index $\left(\mathrm{kg} / \mathrm{m}^{2}\right)$ & $21.7(3.1)$ \\
Duration of surgery $(\mathrm{h})$ & $10.4(2.3)$ \\
ASA status $(1 / 2 / 3)$ & $0 / 12 / 8$ \\
RA solution $(\mathrm{mL} / \mathrm{kg})$ & $58.0(12.4)$ \\
HES solution $(\mathrm{mL} / \mathrm{kg})$ & $15.6(9.3-18.7)$ \\
PRBC $(\mathrm{mL} / \mathrm{kg})$ & $5.9(0-10.4)$ \\
Urine output $(\mathrm{mL} / \mathrm{kg})$ & $9.8(3.7)$ \\
Blood loss $(\mathrm{mL} / \mathrm{kg})$ & $15.4(12.0-26.6)$ \\
\hline
\end{tabular}

Data are presented as mean (SD) or median (interquartile range) depending on distribution. RA: Ringer's acetate; HES: hydroxyethyl starch; PRBC: packed red blood cells. 
$=61,3.1[1.1]$ times per patient; HES: $\mathrm{n}=62,3.1$ [1.0] times per patient). There were no significant differences between RA and HES boluses with respect to time to bolus infusion, urine volume during a 1-hour period, and pre-bolus values of MAP, HR, SVV, SVI, and $\Delta V_{\mathrm{ECF}}$ (Table 2). $\triangle \mathrm{SVV}$ between RA and HES boluses was significantly different at $15 \mathrm{~min}(0.08 \%$ vs. $-0.21 \%, P=$ $0.0075)$ and $30 \mathrm{~min}(0.19 \%$ vs. $-0.21 \%, P=0.048)$. $\triangle$ SVI between RA and HES boluses was also significantly different at $15 \min (-0.33 \%$ vs. $0.59 \%, P=0.010)$. There was no correlation between pre-bolus SVV and pre-bolus $\Delta V_{\mathrm{ECF}}\left(r^{2}=0.017, P=0.15\right)$.

The HES bolus showed a larger $\Delta$ SVI value at $60 \mathrm{~min}$ compared to values at $15 \mathrm{~min}(P=0.034)$ and $30 \mathrm{~min}(P$ $=0.047)$, while there was no difference in $\triangle \mathrm{SVI}$ for the RA bolus $(P=0.78)$.

\subsection{Comparison of Pre-Bolus SVV and $\Delta V_{\mathrm{ECF}}$ between Non-Responding and Responding Fluid Boluses}

There were no differences in pre-bolus SVV between non-responding and responding fluid boluses for RA and HES at 15, 30, and $60 \mathrm{~min}$ after initiating bolus infusion (Figure 2). While the RA bolus did not show significant differences in pre-bolus $\Delta V_{\mathrm{ECF}}$ between non-responding and responding fluid boluses, pre-bolus $\Delta V_{\mathrm{ECF}}$ for the responding HES bolus was larger compared to that of the non-responding HES bolus at $30 \mathrm{~min}(P=0.0072)$ and 60 $\min (P=0.0019)$ after initiating bolus infusion (Figure 3).

\subsection{Prediction of Fluid Responsiveness}

Receiver operating characteristic curves for predicting fluid responsiveness for RA and HES boluses by prebolus SVV and pre-bolus $\Delta V_{\mathrm{ECF}}$ are shown in Figures 4 and $\mathbf{5}$, respectively.

The best AUCs for identifying fluid responsiveness were seen with pre-bolus $\Delta V_{\mathrm{ECF}}$ for HES at $30 \mathrm{~min}$ and $60 \min (\mathrm{AUC}=0.74[0.08], P=0.022 ; \mathrm{AUC}=0.74$ [0.07], $P=0.0054$, respectively). Optimal threshold values of pre-bolus $\Delta V_{\mathrm{ECF}}$ for predicting fluid responsiveness were $6.5 \%$ for $30 \mathrm{~min}$ with a sensitivity of $78 \%$

Table 2. Comparison of hemodynamic parameters between bolus infusions of Ringer's acetate (RA) and hydroxyethyl starch (HES) solutions.

\begin{tabular}{|c|c|c|c|c|}
\hline Variable & RA bolus $(\mathrm{N}=61)$ & HES bolus $(\mathrm{N}=62)$ & Mean Difference ${ }^{*}$ & $P$-value \\
\hline Time to bolus infusion (h) & $4.9(2.9)$ & $5.0(2.9)$ & -1.0 to 1.0 & 0.97 \\
\hline Urine volume $(\mathrm{mL} / \mathrm{kg})^{\dagger}$ & $0.87(0.64)$ & $0.86(0.51)$ & -0.19 to 0.22 & 0.91 \\
\hline Blood loss (mL/kg) & $2.2(1.9)$ & $2.4(2.4)$ & -0.89 to 0.66 & 0.78 \\
\hline $\operatorname{PRBC}(\mathrm{mL} / \mathrm{kg})^{\dagger}$ & $0.79(1.59)$ & $0.79(1.58)$ & -0.56 to 0.56 & 0.99 \\
\hline Vasopressor use (times) ${ }^{\#}$ & $0.8(1.4)$ & $0.7(1.1)$ & -0.4 to 0.5 & 0.79 \\
\hline \multicolumn{5}{|l|}{ Pre-bolus values } \\
\hline MAP (mmHg) & $72(15)$ & $71(14)$ & -3 to 7 & 0.48 \\
\hline HR (beats/minute) & $77(16)$ & $81(16)$ & -9 to 2 & 0.20 \\
\hline SVV (\%) & $13.2(5.3)$ & $13.0(5.1)$ & -1.6 to 2.0 & 0.82 \\
\hline $\mathrm{SVI}\left(\mathrm{mL} / \mathrm{m}^{2}\right)$ & $37.4(7.2)$ & $37.0(6.9)$ & -2.1 to 2.9 & 0.77 \\
\hline$\Delta V_{\mathrm{ECF}}(\%)$ & $6.5(4.8)$ & $6.2(4.4)$ & -1.3 to 1.9 & 0.72 \\
\hline$\Delta \mathrm{SVV}$ at $15 \min (\%)$ & $0.08(0.55)$ & $-0.21(0.62)$ & 0.08 to 0.49 & 0.0075 \\
\hline at $30 \min (\%)$ & $0.19(1.10)$ & $-0.21(1.11)$ & 0.00 to 0.79 & 0.048 \\
\hline at $60 \mathrm{~min}(\%)$ & $0.01(2.08)$ & $-0.39(2.02)$ & -0.32 to 1.12 & 0.28 \\
\hline$\Delta \mathrm{SVI}$ at $15 \min (\%)$ & $-0.33(1.92)$ & $0.59(1.96)^{\$}$ & -1.60 to -0.23 & 0.010 \\
\hline at $30 \min (\%)$ & $-0.41(3.59)$ & $0.86(4.05)^{\$}$ & -2.63 to 0.08 & 0.067 \\
\hline at $60 \min (\%)$ & $-0.06(7.08)$ & $1.95(7.55)$ & -4.60 to 0.57 & 0.13 \\
\hline
\end{tabular}

Data are presented as mean (SD) or median (interquartile range). MAP: mean arterial blood pressure; HR: heart rate; SVV: stroke volume variation; SVI: stroke volume index; $\Delta V_{\mathrm{ECF}}$ : percent change of extracellular fluid volume relative to that at skin incision; $\Delta \mathrm{SVV}$ : change of SVV after start of bolus infusion relative to pre-bolus; $\triangle$ SVI: change of SVI after start of bolus infusion relative to pre-bolus. ${ }^{*} 95 \%$ confidence interval; ${ }^{\dagger}$ during a 1 -hour period from the start of bolus infusion; " intravenous administration of ephedrine or phenylephrine during a $15-60$ min period after the start of bolus infusion; ${ }^{\$}$ difference compared to 60 min $(P<0.05)$. No vasopressor was used during the $0-15 \mathrm{~min}$ period after the start of bolus infusion according to the protocol. Time to bolus infusion denotes hours from the time of skin incision to the time when the fluid bolus was initiated. 

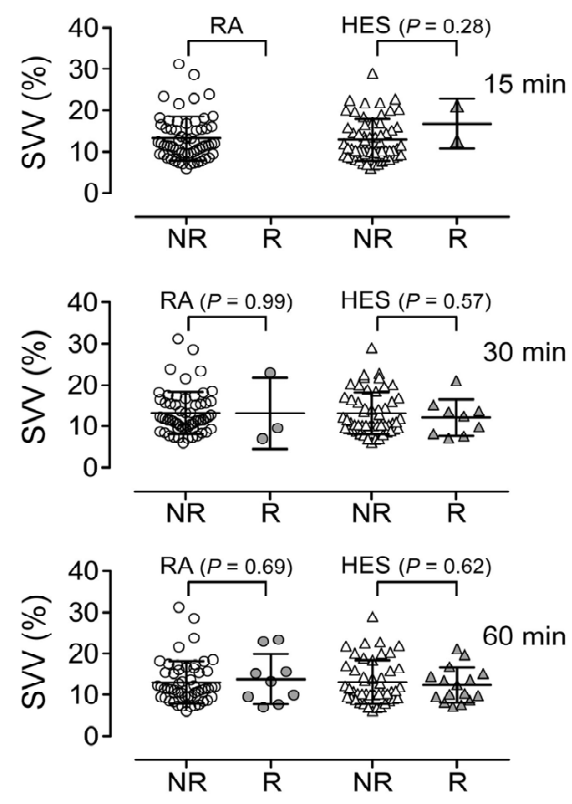

Figure 2. Comparison of pre-bolus stroke volume variation (SVV) between non-responding (NR) and responding (R) fluid boluses for Ringer's acetate $(R A, n=61)$ or hydroxyethyl starch $($ HES, $n=62)$ at 15,30 , and 60 min after initiating bolus infusion. Data are presented as mean (SD).

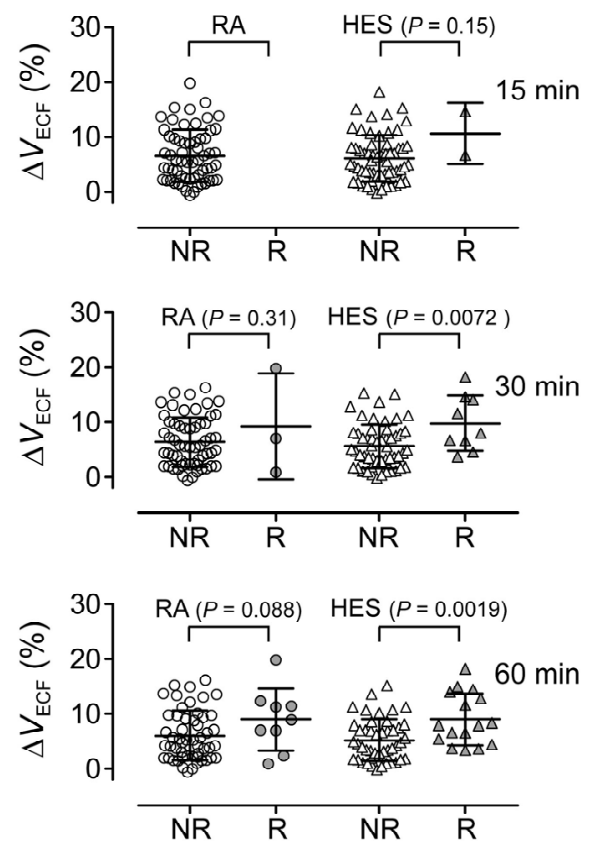

Figure 3. Comparison of pre-bolus percent changes of extracellular fluid volume relative to that at skin incision $\left(\Delta V_{E C F}\right)$ between non-responding (NR) and responding (R) fluid boluses for Ringer's acetate $(R A, n=61)$ or hydroxyethyl starch $(\mathrm{HES}, \mathrm{n}=62)$ at 15,30 , and $60 \mathrm{~min}$ after initiating bolus infusion. Data are presented as mean (SD).

(95\% CI, $40 \%$ to $97 \%$ ) and a specificity of $58 \%$ (95\% CI, $44 \%$ to $72 \%$ ), and $7.7 \%$ for 60 min with a sensitivity of
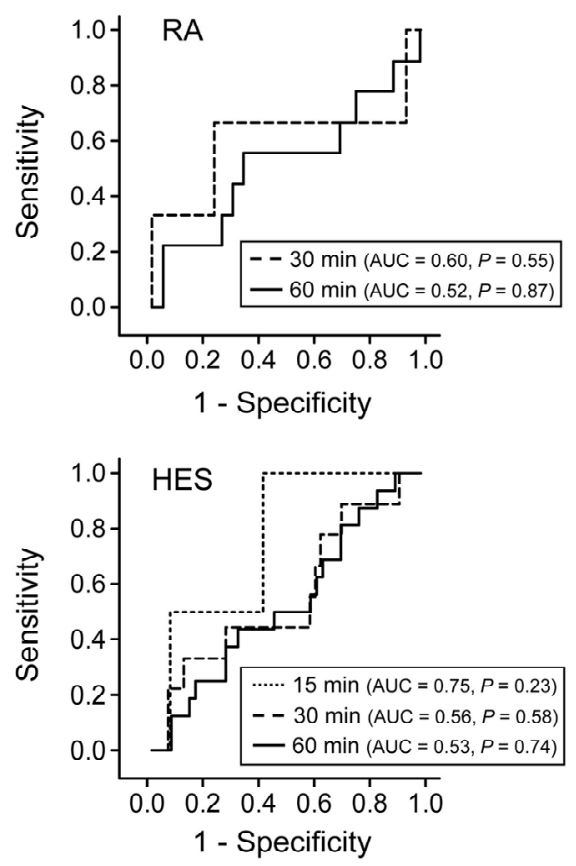

Figure 4. Receiver operating characteristic curve showing the ability of pre-bolus stroke volume variation to predict fluid responsiveness for Ringer's acetate $(\mathrm{RA}, \mathrm{n}=61)$ or hydroxyethyl starch (HES, $n=62$ ). AUC: area under the receiver operating characteristic curve.
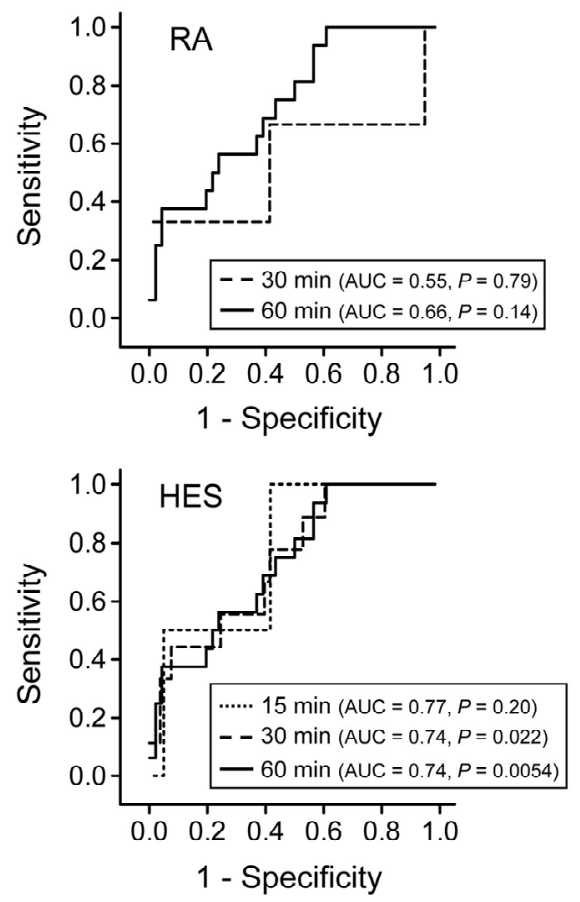

Figure 5. Receiver operating characteristic curve showing the ability of pre-bolus percent changes of extracellular fluid volume relative to that at skin incision to predict fluid responsiveness for Ringer's acetate $(\mathrm{RA}, \mathrm{n}=61)$ or hydroxyethyl starch (HES, $n=62)$. AUC: area under the receiver operating characteristic curve. 
$56 \%(95 \% \mathrm{CI}, 30 \%$ to $80 \%)$ and a specificity of $76 \%$ (95\% CI, 61\% to $87 \%$ ).

\section{Discussion}

The major finding of the present study was that the responding fluid bolus for HES showed a larger pre-bolus $\Delta V_{\mathrm{ECF}}$ compared to the non-responding fluid bolus. The optimal threshold value of pre-bolus $\Delta V_{\mathrm{ECF}}$ for predicting fluid responsiveness after HES bolus infusion was $6 \%$ $8 \%$.

A comparison of cardiac preload change (i.e., $\triangle \mathrm{SVV}$ ) and fluid responsiveness (i.e., $\Delta \mathrm{SVI}$ ) between RA and HES boluses was not the primary aim of this study. The RA bolus served as a control for the HES bolus given that values of $\triangle \mathrm{SVV}$ and $\Delta \mathrm{SVI}$ are affected by not only the type of fluid solution but also various factors such as surgical stress and blood loss. Fluid bolus loading was conducted based on urine output. While urine output is not a validated criterion for fluid bolus during surgery, urine output is a routinely used clinical parameter for deciding on fluid bolus loading [15]. Moreover, a SVVguided fluid bolus (e.g., SVV > 13\%) may be inappropriate for this study because it would result in a narrow range of pre-bolus SVV.

Contrary to our expectations, pre-bolus SVV did not predict fluid responsiveness after HES bolus infusion. The threshold of SVV for fluid responsiveness defined as an increase in cardiac output (e.g., 12\%) was reported to range from $9.5 \%$ to $12.5 \%$ [16]. Despite that the mean value of pre-bolus SVV for HES bolus infusions in the present study (13\%) falls within this range, HES bolus infusion on average caused only a minimal SVV decrease $(0.2 \%)$ and SVI increase $(0.6 \%)$ at the end of fluid infusion. This SVV decrease was much smaller compared to the $3 \% \mathrm{SVV}$ decrease after a $250 \mathrm{~mL}$ bolus infusion of HES 130/0.4 solution during major abdominal surgery in another study [17]. Based on this, we defined fluid responsiveness as an increase in SVI of $\geq 5 \%$, which is smaller than values usually used (e.g., 10\%) [18]. Indeed, ongoing blood loss $(2.4 \mathrm{~mL} / \mathrm{kg}$ on average $)$ may be responsible for the small increase in cardiac preload after HES bolus infusion and substantial fluid unresponsiveness in our study. However, capillary leakage due to surgical injury may also be involved in that it can lead to poor plasma volume expansion [19]. This possibility is supported by the finding that HES was infused at $5.7 \mathrm{~h}$ on average after skin incision and the peak of microvascular permeability at the surgical site begins at 3 or 4 hours after surgical injury [20].

No correlation was found between pre-bolus SVV and pre-bolus $\Delta V_{\mathrm{ECF}}$ ranging from $0.1 \%$ to $16 \%\left(r^{2}=0.017\right)$, suggesting that fluid accumulates in the extracellular fluid space independently of intravascular volume status.
For HES, we found a larger pre-bolus $\Delta V_{\mathrm{ECF}}$ in responding boluses compared to non-responding boluses at 30 min and 60 min after initiating bolus infusion (Figure 3). Given that bioelectrical resistance at zero frequency reflects extracellular fluid volume changes mainly in the interstitium [11-13,21], this finding suggests that HES enhances intravascular volume expansion by extracting accumulated fluid from the interstitium to the intravascular space due to a colloid osmotic effect. This increases in intravascular volume by HES may have increased cardiac preload, thereby increasing stroke volume in the steep portion of the Frank-Starling curve. This scenario is consistent with a previous study in healthy volunteers showing that gelatin infusion increased blood volume, while it decreased extracellular fluid volume as assessed by the conductivity technique [22]. This scenario is also supported by the significantly large $\Delta$ SVI at $60 \mathrm{~min}$ compared to those at $15 \mathrm{~min}$ and $30 \mathrm{~min}$ after initiating HES bolus infusion (Table 2), suggesting that this fluid shift takes up to an hour to complete.

The validity of uncalibrated cardiac output measurement using Vigileo-FloTrac system has not yet been fully established. However, two recent studies showed that the third-generation Vigileo-FloTrac device, which was also used in this study, demonstrated improved precision of cardiac output measurement compared to the previous version, and could accurately track changes in cardiac output in anesthetized patients [23,24]. Longitudinal analysis of fluid bolus infusion is another problem in our study because the effects of each infusion on SVV and SVI changes were not completely independent. Moreover, SVI values are affected by the balance of surgical stress and depth of anesthesia, which may differ depending on the surgical procedure. However, given that these problems are common to RA and HES boluses, a significantly large pre-bolus $\Delta V_{\mathrm{ECF}}$ for responding boluses compared to non-responding boluses for HES suggests that fluid responsiveness after HES bolus infusion depends on extracellular fluid volume. Finally, we used bioelectrical impedance to assess extracellular fluid volume. Given that bioelectrical impedance electrically measures extracellular fluid volume, absolute value of extracellular fluid volume predicted by this method is not identical with anatomical value of extracellular fluid volume [11]. However, as relative change of bioelectrical impedance in the same patient could detect time-course of extracellular fluid accumulation in surgical patients [11-13], this problem is unlikely to affect our conclusion.

Goals for fluid optimization, such as cardiac output and stroke volume, mainly relate to intravascular volume but provide no information on extracellular fluid volume. Our study showed that increased cardiac preload (i.e., decrease in SVV) at the end of HES bolus infusion may 
be reduced due to capillary leakage arising from surgical injury. Yet, upon enhanced accumulation of extracellular fluid, HES bolus infusion can recover cardiac preload and fluid responsiveness 30 - 60 min after bolus infusion by extracting more fluid from the interstitial space. The threshold of pre-bolus $\Delta V_{\mathrm{ECF}}$ for exerting this effect was $6 \%-8 \%$, corresponding to $12-16 \mathrm{~mL} / \mathrm{kg}$ of extracellular fluid accumulation in the entire body. The dependence of fluid responsiveness after HES bolus infusion on extracellular fluid volume should be taken into account when determining the optimal timing and right volume of HES infusion for intra- and postoperative use in patients undergoing major abdominal surgery. Our findings suggest that HES use is preferable during the later stages of surgery when interstitial fluid accumulates, thereby preventing HES overdose.

In conclusion, our finding suggests that extracellular fluid volume can predict fluid responsiveness after HES bolus infusion during major abdominal surgery, which becomes larger with interstitial fluid accumulation.

\section{Acknowledgements}

We thank Dr. Maxime Cannesson for critical comments and valuable suggestions. This work was supported by Grants-in-Aid for Scientific Research (C) [No. 20591846] and [No. 24592365] from the Ministry of Education, Science and Culture of Japan. Tsuneo Tatara received speaking fees from Edwards Lifesciences and Fresenius Kabi Japan.

\section{REFERENCES}

[1] T. J. Gan, A. Soppitt, M. Maroof, et al., "Goal-Directed Intraoperative Fluid Administration Reduces Length of Hospital Stay after Major Surgery," Anesthesiology, Vol. 97, No. 4, 2002, pp. 820-826. http://dx.doi.org/10.1097/00000542-200210000-00012

[2] A. Donati, S. Loggi, J.-C. Preiser, et al., "Goal-Directed Intraoperative Therapy Reduces Morbidity and Length of Hospital Stay in High-Risk Surgical Patients," Chest, Vol. 132, No. 6, 2007, pp. 1817-1824. http://dx.doi.org/10.1378/chest.07-0621

[3] M. Bundgaard-Nielsen, K. Holte, N. H. Secher and H. Kehlet, "Monitoring of Peri-Operative Fluid Administration by Individualized Goal-Directed Therapy," Acta Anaesthesiologica Scandinavica, Vol. 51, No. 3, 2007, pp. 331-340. http://dx.doi.org/10.1111/j.1399-6576.2006.01221.x

[4] C. S. Hartog, M. Bauer and K. Reinhart, "The Efficacy and Safety of Colloid Resuscitation in the Critically Ill," Anesthesia \& Analgesia, Vol. 112, No. 1, 2011, pp. 156164. http://dx.doi.org/10.1213/ANE.0b013e3181eaff91

[5] C. S. Hartog, M. Kohl and K. Reinhart, "A Systematic Review of Third-Generation Hydroxyethyl Starch (HES130/ 0.4 ) in Resuscitation: Safety Not Adequately Addressed,"
Anesthesia \& Analgesia, Vol. 112, No. 3, 2011, pp. 635645. http://dx.doi.org/10.1213/ANE.0b013e31820ad607

[6] D. Chappell, M. Jacob, K. Hofmann-Kiefer, et al., "A Rational Approach to Perioperative Fluid Management," Anesthesiology, Vol. 109, No. 4, 2008, pp. 723-740. http://dx.doi.org/10.1097/ALN.0b013e3181863117

[7] J.-L. Vincent, "Let's Give Some Fluid and See What Happens" versus the "Mini-Fluid Challenge," Anesthesiology, Vol. 115, No. 3, 2011, pp. 455-456.

http://dx.doi.org/10.1097/ALN.0b013e318229a521

[8] M. Cannesson, "Non-Invasive Guidance of Fluid Therapy," In: R. G. Hahn, Ed., Clinical Fluid Therapy in the Perioperative Setting, Cambridge University Press, Cambridge, 2011, pp. 103-111. http://dx.doi.org/10.1017/CBO9780511733253

[9] T. Tatara, Y. Nagao and C. Tashiro, "Effect of Duration of Surgery on Fluid Balance during Abdominal Surgery: A Mathematical Model," Anesthesia \& Analgesia, Vol. 109, No. 1, 2009, pp. 211-216. http://dx.doi.org/10.1213/ane.0b013e3181a3d3dc

[10] K. Aukland and R. K. Reed, "Interstitial-Lymphatic Mechanisms in the Control of Extracellular Fluid Volume," Physiological Reviews, Vol. 73, No. 1, 1993, pp. 1-78.

[11] T. Tatara and K. Tsuzaki, "Segmental Bioelectrical Impedance Analysis Improves the Prediction for Extracellular Water Volume Changes during Abdominal Surgery," Critical Care Medicine, Vol. 26, No. 3, 1998, pp. 470476.

http://dx.doi.org/10.1097/00003246-199803000-00017

[12] D. Bracco, J.-P. Revelly, M. M. Berger and R. Chioléro, "Bedside Determination of Fluid Accumulation after Cardiac Surgery Using Segmental Bioelectrical Impedance," Critical Care Medicine, Vol. 26, No. 6, 1998, pp. 10651070.

http://dx.doi.org/10.1097/00003246-199806000-00029

[13] D. Bracco, M. M. Berger, J.-P. Revelly, et al., "Segmental Bioelectrical Impedance Analysis to Assess Perioperative Fluid Changes," Critical Care Medicine, Vol. 28, No. 7, 2000, pp. 2390-2396. http://dx.doi.org/10.1097/00003246-200007000-00034

[14] A. De Lorenzo, A. Andreoli, J. Matthie and P. Withers, "Predicting Body Cell Mass with Bioimpedance by Using Theoretical Methods: A Technological Review," Journal of Applied Physiology, Vol. 82, No. 5, 1997, pp. 15421558.

[15] V. Nisanevich, I. Felsenstein, G. Almogy, et al., "Effect of Intraoperative Fluid Management on Outcome after Intraabdominal Surgery," Anesthesiology, Vol. 103, No. 1, 2005, pp. 25-32.

http://dx.doi.org/10.1097/00000542-200507000-00008

[16] J. Renner, J. Scholz and B. Bein, "Monitoring Fluid Therapy," Best Practice \& Research Clinical Anaesthesiology, Vol. 23, No. 2, 2009, pp. 159-171. http://dx.doi.org/10.1016/j.bpa.2008.12.001

[17] D. Lahner, B. Kabon, C. Marschalek, et al., "Evaluation of Stroke Volume Variation Obtained by Arterial Pulse Contour Analysis to Predict Fluid Responsiveness Intraoperatively," British Journal of Anaesthesia, Vol. 103, 
No. 3, 2009, pp. 346-351.

http://dx.doi.org/10.1093/bja/aep200

[18] T. E. Miller and T. J. Gan, "Goal-Directed Fluid Therapy," In: R. G. Hahn, Ed., Clinical Fluid Therapy in the Perioperative Setting, Cambridge University Press, Cambridge, 2011, pp. 91-102.

http://dx.doi.org/10.1017/CBO9780511733253.012

[19] J. Persson and P.-O. Grände, "Plasma Volume Expansion and Transcapillary Fluid Exchange in Skeletal Muscle of Albumin, Dextran, Gelatin, Hydroxyethyl Starch, and Saline after Trauma in the Cat," Critical Care Medicine, Vol. 34, No. 9, 2006, pp. 2456-2462.

http://dx.doi.org/10.1097/01.CCM.0000233876.87978.A B

[20] J. C. Fantone and P. A. Ward, "Inflammation," In: E. Rubin and J. L. Farber, Eds., Pathology, Lippincott-Raven Publishers, Philadelphia, 1999, pp. 37-75.

[21] B. T. Tedner, H. S. Jacobson, D. Linnarsson and L. E. Lins, "Impedance Fluid Volume Monitoring during In- travenous Infusion in Healthy Subjects," Acute Care, Vol. 10, No. 3-4, 1983, pp. 200-206.

[22] C. G. Olthof, J. P. P. M. de Vries, P. M. J. M. de Vries, et al., "The Influence of Ringer's Lactate and Gelatin Infusion on the Internal Fluid Balance of Healthy Volunteers Measured by a Non-Invasive Conductivity Technique," European Journal of Anaesthesiology, Vol. 10, No. 6, 1993, pp. 397-402.

[23] G. Biancofiore, L. A. H. Critchley, A. Lee, et al., "Evaluation of a New Software Version of the Flotrac/Vigileo (Version 3.02) and a Comparison with Previous Data in Cirrhotic Patients Undergoing Liver Transplant Surgery," Anesthesia \& Analgesia, Vol. 113, No. 3, 2011, pp. 515522.

[24] L. Meng, N. P. Tran, B. S. Alexander, et al., "The Impact of Phenylephrine, Ephedrine, and Increased Preload on Third-Generation Vigileo-FloTrac and Esophageal Doppler Cardiac Output Measurements," Anesthesia \& Analgesia, Vol. 113, No. 4, 2011, pp. 751-757. 